\title{
Analysis of microscopic magnitudes of radiative blast waves launched in xenon clusters with collisional-radiative steady-state simulations
}

\author{
R. Rodriguez ，G. Espinosa , J.M. Gil , R. Florido ， J.G. Rubiano \\ M.A. Mendoza , P. Martel , E. Minguez , D.R. Symes , \\ M. Hohenberger , R.A. Smith
}

\begin{abstract}
A B S T R A C T
Radiative shock waves play a pivotal role in the transport energy into the stellar medium. This fact has led to many efforts to scale the astrophysical phenomena to accessible laboratory conditions and their study has been highlighted as an area requiring further experimental investigations. Low density material with high atomic mass is suitable to achieve radiative regime, and, therefore, low density xenon gas is commonly used for the medium in which the radiative shocks such as radiative blast waves propagate. In this work, by means of collisionalradiative steady-state calculations, a characterization and an analysis of microscopic magnitudes of laboratory blast waves launched in xenon clusters are made. Thus, for example, the average ionization, the charge state distribution, the cooling time or photon mean free paths are studied. Furthermore, for a particular experiment, the effects of the self-absorption and self-emission in the specific intensity emitted by the shock front and that is going through the radiative precursor are investigated. Finally, for that experiment, since the electron temperature is not measured experimentally, an estimation of this magnitude is made both for the shock shell and the radiative precursor.
\end{abstract}

\section{Introduction}

The popularity of the field of laboratory astrophysics has grown considerably over the last two decades. Two developments in the field have contributed to the successful design of laboratory astrophysical models: first, it has been demonstrated that the hydrodynamics can be scaled correctly between laboratory and astrophysical scenarios [1-6]; second, the improvement of high-power laser systems allows us to generate plasmas that are in the regimes for certain astrophysical systems. Those experiments permit to explain and predict what occurs in astrophysical phenomena and have the advantage of being repeatable and that the initial conditions are under control. Besides, those experiments also provide important data for verification and validation of several aspects of numerical codes such as atomic physics, equation of state, radiative transfer and hydrodynamics.

One of the most interesting astrophysical phenomena is the shock waves which are ubiquitous throughout the universe and play a crucial role in the transport of energy into the interstellar medium [7]. When the radiation transport is important to the total energy budget, shock waves can be radiatively driven so that its dynamics can be 
significantly modified by radiative processes. At high shock velocity, the shocked medium is heated and ionized emitting radiation, which gives rise to radiative cooling. The radiation emitted in turn heats and ionizes the unshocked medium leading to the creation of a radiative precursor [8]. Radiative shocks are observed around astronomical objects in a wide variety of forms, e.g. accretion shock, pulsating stars, supernovae in their radiative cooling stage, bow shocks of stellar jet in galactic medium, collision of interstellar clouds and entry of rockets or comets into planetary atmospheres [9-11] and they are also observed in laser inertial fusion [12].

Laboratory studies of radiative shocks are, currently, a research area of interest thanks to the advent of experimental facilities that are able to produce high energy-density conditions. Thus, in laboratory, three methods have been commonly used to create radiative shocks in gases [13] and they can be characterized according to the optical depth of the gas ahead of (upstream) and behind (downstream) the shock. The first is to make a shock tube [14] by driving a solid density plastic or beryllium piston into a xenon gas cell. These experiments have been conducted at Omega [3,15-18] and LULI [19-22] using $\mathrm{kJ}$ lasers obtaining planar geometry. Because the laser pulse is long $(\approx n s)$ there is a continuous injection of energy to the shock which produces a stationary structure. The shock speeds reached in this kind of experiments are very high $\left(>50 \mathrm{~km} \mathrm{~s}^{-1}\right)$ and the shocked gas is highly compressed $(>30)$ due to the strong radiative losses. Because of the high density of the piston the downstream medium is optically thick whereas the upstream medium is optically thin, and for this reason this system is classified as thick-thin [23]. An astrophysical example of this kind of systems is the accretion shocks produced in some binary systems.

A different type of shock is produced after a sudden release of energy in a zero-extension and instantaneous explosion. In this case, a shock moves into the surrounding medium creating a blast wave, that is generally described as an expanding shock that is in the process of sweeping up the material that is ahead of the shock. The blast waves have been generated experimentally in two alternative ways. In the first one, by using a kJ laser to irradiate a pin or foil within a moderate to high $Z$ background gas $[24,25]$. In the second one, the laser energy can be deposited directly into gas formed by atomic clusters to launch shocks [7,26-36]. Clustered gases exhibit extremely efficient absorption of intense laser light creating a hot, high energy density plasma in a low average density target. This plasma subsequently explodes into the ambient gas forming a cylindrical blast wave [26]. Thus, high Mach number shocks can be launched using high intensity lasers ( $>10^{17}$ $\mathrm{W} \mathrm{cm}{ }^{-2}$ ) with energies lower than $1 \mathrm{~J}$. For a given shock velocity and a given initial gas pressure, materials with medium or high atomic numbers suit the achievement of the radiative regime, and for this reason, krypton and xenon are commonly used for the medium in which the radiative shock propagates. In these low density gas blast waves both the upstream and downstream medium are optically thin and therefore they can be classified as thin-thin [37]. Thin-thin shocks are the most commonly observed in astrophysics. Supernova remnant shocks in dense enough environment are of this type, for example.
In this work an analysis of some microscopic properties of radiative blast waves launched in xenon clusters is made. In particular, their thermodynamic regimes, the photon mean free paths (PMFP), the cooling times, the average ionization and the charge state distributions (CSDs) are studied. This analysis will allow us to characterize the laboratory blast waves launched in xenon clusters. Furthermore, for a particular experiment carried out using the THOR laser system at the University of Texas [33], the influence of the self-absorption and the selfemission is studied, both in the calculation of the specific intensity emitted by the shock front and which is going through the radiative precursor. Furthermore, as the electron temperature of the plasma is not experimentally measured, an estimation of this magnitude is also made both for the shock shell and the radiative precursor. To perform this analysis kinetics calculations under stationary approach were made using the computational package ABAKO/RAPCAL [38]. The next section is devoted to a brief description of this computational package. In Section 3 the analysis of the microscopic magnitudes is performed and finally in the last section conclusions and general remarks are presented.

\section{Theoretical model}

The calculations in this work were performed using the computational package ABAKO/RAPCAL [38] that consists of two codes, ABAKO [39] and RAPCAL [40].

\section{1. $A B A K O$}

ABAKO is devoted to the calculation of the plasma level populations using a collisional-radiative steady-state (CRSS) model. The CRSS model is solved level by level (or configuration by configuration, depending on the atomic description used) and it is applied to low-to-high $Z$ ions under a wide range of plasma conditions: Coronal equilibrium, local and non-local thermodynamic equilibrium (LTE and NLTE, respectively), optically thin and thick plasmas. Following the standard NLTE modeling approach, where an account of the existing atomic states is made and the microscopic (radiative and collisional) processes connecting these states are identified, a rate equation system describing the population density of the atomic states is built and solved, giving the population distribution. Therefore, to find the level population distribution, under stationary situations, the following system of rate equations is solved:

$\sum_{\zeta^{\prime} j} N_{\zeta^{\prime} j} \mathbb{R}_{\zeta^{\prime} j \rightarrow \zeta i}^{+}-\sum_{\zeta^{\prime} j} N_{\zeta i} \mathbb{R}_{\zeta^{-} \rightarrow \zeta^{\prime} j}^{-}=0$,

where $N_{\zeta i}$ is the population density of the atomic level $i$ of the ion with charge state $\zeta$. The terms $\mathbb{R}_{\zeta j \rightarrow \zeta i}^{+}$and $\mathbb{R}_{\zeta^{\prime} j \rightarrow \zeta i}$ take into account all the atomic processes which contribute to populate and depopulate the state $\zeta i$, respectively. This set of equations constitutes the so-called CRSS model. In this work, the calculations of the plasma atomic level populations were performed using the CRSS implemented in the computational code named ABAKO [39]. In ABAKO it is assumed that the system has had enough time to thermalize and, therefore, both the electrons and ions have a Maxwell-Boltzmann type 
energy distribution. Furthermore, in ABAKO it is also assumed that electron and ion temperatures are equal. Therefore, in the following, it will denote the plasma temperature by the electron temperature $T_{e}$.

Two complementary equations which have to be satisfied together with (1) are, first, the requirement that the sum of all the partial densities equals the total ion density, $n_{\text {ion }}$,

$$
\sum_{\zeta=0}^{Z} \sum_{i=0}^{M_{\zeta}-1} N_{\zeta i}=n_{\text {ion }}
$$

and, second, the charge neutrality condition in the plasma,

$$
\sum_{\zeta=0}^{Z} \sum_{i=0}^{M_{\zeta}-1} \zeta N_{\zeta i}=n_{e}
$$

where $M_{\zeta}$ is the total number of levels for the charge state $\zeta$ and $n_{e}$ the electron density. The plasma average ionization is defined as

$\bar{Z}=\frac{\sum_{\zeta=0}^{Z} \zeta N_{\zeta}}{\sum_{\zeta=0}^{Z} N_{\zeta}}=\frac{n_{e}}{n_{\text {ion }}}$

and the plasma CSD is defined as the set of the population densities, $\left(N_{\zeta}\right)$, of the ions present in the plasma for a given condition of density and temperature and which is obtained from the resolution of the rate equations. A special care was taken during the development of this CRSS model to achieve an optimal equilibrium between accuracy and computational cost. Hence, it has been employed as analytical expressions for the rate coefficients of the atomic processes included in the CRSS model, which yield a substantial saving of computational requirements, but provide satisfactory results in relation to those obtained from more sophisticated codes and experimental data as it has been proved in some of the last NLTE code comparison workshops [41-43]. The processes included in this CRSS model are the following: collisional ionization [44] and three-body recombination, spontaneous decay, collisional excitation [45] and deexcitation, radiative recombination [46], electron capture and autoionization. Between brackets it has been added with the references from where their approximated analytical rates coefficients have been acquired. The rates of the inverse processes are obtained through the detailed balance principle. It is worth pointing out that the autoionizing states are included explicitly. It has been proved that their contribution is critical in the determination of the ionization balance. The cross section of the autoionization is evaluated using detailed balance principle from the electron capture cross section. This one is obtained from the collisional excitation cross section using a known approximation [47]. In this paper no radiation-driven processes are explicitly considered.

Since the number of rate equations is large due to the number of atomic levels involved, ABAKO makes use of the technique of sparse matrices to store the non-zero elements of the coefficient matrix of the system, which implies substantial savings in memory requirement. For the matrix inversion we use iterative procedures [38] because they entail much less memory than direct methods and they are also faster.
The atomic data employed in this work were obtained from FAC code [48]. Since in this work we are interested in low ionized Xe plasmas, the atomic calculations were carried out in the relativistic detailed configuration accounting (RDCA) approach. The radiative transitions rates in FAC are calculated in the single multipole approximation, and in this work they were obtained in the electric dipole approach. The Unresolved Transition Array (UTA) [49] formalism is used for the bound-bound transitions, so the transition energies include the UTA shift, and the width for each transition is considered. Furthermore, the line strengths are corrected for configuration interaction within the same non-relativistic configurations. The continuum lowering due to the influence of the plasma surrounding is also considered and is calculated by means of the expression due to Stewart and Pyatt [50]. Due to the inclusion of the continuum lowering, the kinetics equations must be solved iteratively, since the atomic data depend on the ionization balance.

A key factor in the CRSS calculations is the choice of configurations included in the model. Including configurations with energies up to three times the ionization potential should be adequate for accurate modeling of thermal plasmas $[51,52]$. Thus, the set of configurations included was selected according to the following criteria: (1) ground configuration; (2) single excited configurations from the valence shell, $n_{v}$, to shells with $n_{v} \leq n \leq n_{v}+4$; (3) doubly excited configurations from the valence shell to shells with $n_{v} \leq n \leq n_{v}+2$ and (4) single excited configurations from the shell $n_{v}-1$ to shells with $n_{v} \leq n \leq n_{v}+2$.

\subsection{RAPCAL}

In this work, the plasma radiative properties were calculated using RAPCAL computational code. This one was developed to obtain several of these quantities such as the monochromatic absorption and emission coefficients, mean and multigroup opacities, source functions, radiative power losses, specific intensities and plasma transmission. A detailed description of the code can be found in [40]. For this work, we focused our interest on the multifrequency and mean opacities, the radiative power losses and the specific intensities. The monochromatic absorption is denoted in this work as $\kappa(\nu)$, and it includes the bound-bound, bound-free and free-free contributions

$\kappa(\nu)=\kappa_{b b}(\nu)+\kappa_{b f}(\nu)+\kappa_{f f}(\nu)$,

where $\nu$ is the photon frequency. The bound-bound contribution to the absorption is given by

$\kappa_{b b}(\nu)=\sum_{\zeta} \sum_{i, j} \kappa_{\zeta i \rightarrow \zeta j}(\nu)$,

with

$\kappa_{\zeta i \rightarrow \zeta j}(\nu)=\frac{h \nu}{4 \pi} N_{\zeta i} \frac{g_{\zeta j}}{g_{\zeta i}} 2 h \nu_{i j}^{3} A_{\zeta j \rightarrow \zeta i} \phi_{i j}(\nu)\left(1-\frac{g_{\zeta i} N_{\zeta j}}{g_{\zeta j}} N_{\zeta i}\right)$,

where $c$ is the speed of light, $g_{\zeta i}$ and $g_{\zeta j}$ are the statistical weights of the $i$ and $j$ levels, respectively, $A_{\zeta j \rightarrow \zeta i}$ is the Einstein coefficient for spontaneous deexcitation [53] between the bound states $j, i$ of the ion $\zeta$ and $h$ is the Planck's constant. In the previous equation, $\phi_{i j}(\nu)$ 
represents the line profile for line absorption. In the evaluation of the line profile, natural, Doppler, UTA and electron-impact [54] broadenings were included. The lineshape function is applied with the Voigt profile that incorporates all these broadenings.

The bound-free contribution to the absorption is given by

$\kappa_{b f}(\nu)=\sum_{\zeta, i} \sum_{\zeta, j} \kappa_{\zeta i \rightarrow \zeta+1 j}(\nu)$

with

$\kappa_{\zeta i \rightarrow \zeta+1 j}(\nu)=N_{\zeta i} \sigma_{\zeta i \rightarrow \zeta+1 j}^{p h o}(\nu)\left(1-\frac{N_{\zeta+1 j} n_{e} f(\varepsilon)}{N_{\zeta i}} \frac{g_{\zeta i}}{g_{\zeta+1 j} g(\varepsilon)}\right)$,

with $\varepsilon$ the energy of the free electron and $m_{e}$ the electron mass. $g(\varepsilon)$ is the density of states with energy $\varepsilon$ which, assuming an ideal gas of free electrons, is given by

$g(\varepsilon)=4 \pi\left(\frac{2 m_{e}}{h^{2}}\right)^{3 / 2} \varepsilon^{1 / 2}$.

As said before, in ABAKO a Maxwell-Boltzmann distribution, $f(\varepsilon)$, is assumed at temperature $T_{e}$ for the free electrons. Since the line contribution is the most relevant in the plasma conditions analyzed, photoionization cross section, $\sigma_{\zeta i \rightarrow \zeta+1 j}^{p h o}(\nu)$ has been evaluated using the semiclassical expression of Kramers [46].

For the free-free contribution to the absorption the Kramers semi-classical expression for the inverse bremsstrahlung cross section has been used [55], which is given by

$\kappa_{f f}(\nu)=\frac{16 \pi^{2} e^{2} h^{2} \alpha}{3 \sqrt{3}\left(2 \pi m_{e}\right)^{3 / 2}} \frac{\overline{Z^{2}} n_{i o n} n_{e}}{T_{e}^{1 / 2}(h \nu)^{3}}\left(1-e^{-h \nu / T_{e}}\right)$.

In order to determine the opacity, $k(\nu)$, it also takes into account the absorption due to the scattering of photons. In RAPCAL this one is approximated using the Thomson scattering cross section [56]

$\kappa_{\text {scatt }}=n_{e} \sigma^{\text {Thom }}$,

with $\sigma^{\text {Thom }}=6.65 \times 10^{-25} \mathrm{~cm}^{2}$. Finally, the opacity is given by

$k(\nu)=\frac{1}{\rho}\left(\kappa(\nu)+\kappa_{\text {scatt }}\right)$.

As said before, RAPCAL also provides the Planck $k_{P}$ and Rosseland $k_{R}$ mean opacities, which are given by [57]

$k_{P}=\int_{0}^{\infty} d \nu \tilde{B}(\nu, T)\left(k(\nu)-\kappa_{\text {scatt }} / \rho\right)$

$\frac{1}{k_{R}}=\int_{0}^{\infty} d \nu \frac{\partial \tilde{B}(\nu, T)}{\partial T} \frac{1}{k(\nu)}$,

where $\tilde{B}(\nu, T)$ is the normalized Planckian function,

$\tilde{B}(\nu, T)=\frac{15}{\pi^{4} T_{e}} \frac{u^{3}}{e^{u}-1}, \quad u=\frac{h \nu}{T}$.

The radiative power loss is evaluated as following [58] in $(\mathrm{eV} / \mathrm{s} / \mathrm{ion})$. For the bound-bound contribution

$P_{b b}=\sum_{\zeta} \sum_{i j} h \nu_{i j} A_{i j} N_{\zeta j}$.
The bound-free contribution is given by

$P_{b f}=4 \pi \sum_{\zeta} \sum_{i j} N_{\zeta i}\left(\frac{N_{\zeta+1 j} N_{\zeta}}{N_{\zeta i} N_{\zeta+1}}\right)^{L T E} \int_{\nu_{0}}^{\infty} \sigma_{\zeta i \rightarrow \zeta+1 j}^{p h o}(\nu)\left(\frac{2 h \nu^{3}}{c^{2}}\right) e^{-h \nu / T_{e}} d \nu$,

where $\nu_{0}$ is the threshold energy and the LTE population ratio is obtained from Saha equation. The contribution from the free-free transitions is given for a pure Coulomb field in (eV/s/ion) as following [59]:

$P_{f f}=9.55 \times 10^{-14} n_{e} T_{e}^{1 / 2} \sum_{\zeta} Z_{\zeta}^{2} N_{\zeta}$

where it has been assumed the gaunt factor equal to unity. The total radiative power loss is then obtained as the sum of the three contributions.

\section{Results}

\subsection{Characterization of the blast waves launched in xenon clusters}

In this work we have studied blast waves launched in xenon clusters that typically have a gas density $\approx 5 \times$ $10^{-4} \mathrm{~g} \mathrm{~cm}^{-3}$ and post-shock temperatures $5-20 \mathrm{eV}$. In previous works $[60,61]$ we presented the map of the thermodynamic regimes, i.e. the regions of electron temperature and density of matter in which NLTE or LTE can be assumed, and the map of the average ionization for Xe plasmas in a wide range of plasma densities and temperatures. In this work we have focused our attention in the range of densities and temperatures of interest in this kind of blast waves and the corresponding maps are shown in Fig. 1. To obtain the map of the thermodynamic regimes, we proceeded as follows: when the ion populations, $p_{i}$, calculated from Saha-Boltzmann (SB) equations, $p_{i}^{S B}$, present a mean deviation $(\Delta p)$, with respect to those obtained from the CRSS model, $p_{i}^{C R S S}$, smaller than a certain criterion imposed $\left(\Delta p^{*}\right)$ then we consider that the use of LTE is acceptable for obtaining the ionization balance. The mean deviation is calculated as

$\Delta p^{S B}=\sqrt{\frac{\sum_{i}\left(p_{i}^{C R S}-p_{i}^{S B}\right)^{2}}{\sum_{i}\left(p_{i}^{S B}\right)^{2}}} \leq \Delta p^{*}$,

where $i$ runs over the whole set of ions included in the calculations. The criterion, $\Delta p^{*}$, was fixed to $0.1 \quad(=10 \%)$. In Ref. [61] we showed that taking the value of the criterion equal to 0.1 ensures that the deviations in the average ionization were always less than or equal to $1 \%$ and we also obtained that when the criterion is fulfilled the deviations for the Rosseland and Planck mean opacities are lower than $10 \%$. From the average ionization map we can observe that, for the plasma conditions involved in this kind of experiments, the ionization is small. Thus, from Fig. 2, where we show the CSD as a function of the temperature for a density of matter of $5 \times 10^{-4} \mathrm{~g} \mathrm{~cm}^{-3}$, it is detected that the most ionized ion with significant contribution to the CSD is $\mathrm{Xe}^{+11}$. This low ionization justifies the use of the RDCA in the atomic model.

In Ryutov et al. [1] the conditions to define an optically thin, radiative regime for laboratory astrophysics experiments relevant to radiative supernova remnants were established. In order to define hydrodynamics parameters 

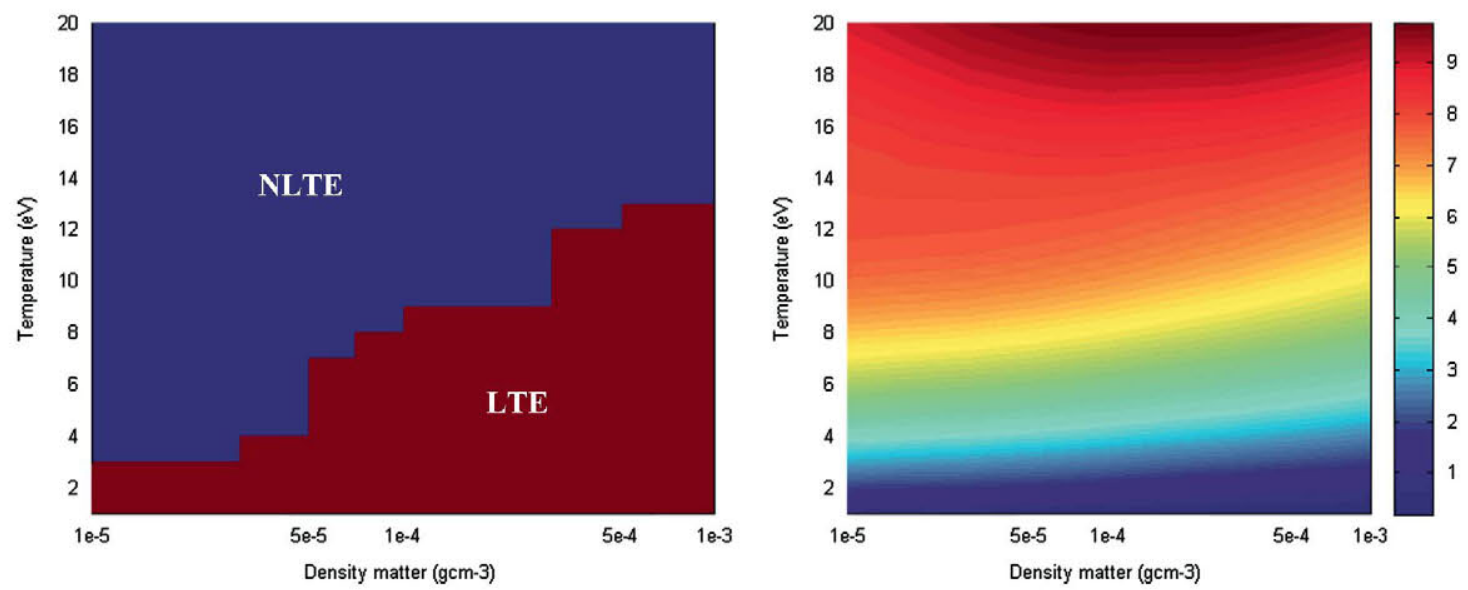

Fig. 1. Xenon thermodynamic regimes (left) and average ionization (right) as a function of electron temperature and density of matter.

such as the shock velocity to reach the radiative shock conditions, one should compare the radiative flux and pressure to the corresponding thermal quantities [20]. Thus, it can be defined as the radiative flux regime as the situation in which the radiative energy flux, $F_{r a d}$, is greater than the material energy flux, $F_{m}$. In this region, thermal and radiative regimes coexist. At much higher temperatures the radiation dominated region is reached where the radiation pressure, $P_{\text {rad }}$, is larger than the material pressure, $P_{m}$. In this second region the plasma is fully dominated by the radiation. Finally, there is a third region in which both material magnitudes are greater than the radiative ones in which the plasma is dominated by thermal phenomena and then the shock behaves purely hydrodynamically. In [62] it was shown that the radiative shock conditions can be more easily achieved by propagating the shock in a low density medium having a high atomic number. These are the reasons why in the experiments considered in this work clusters of xenon are used, with low average density, as the medium for the radiative shock propagates. Furthermore, the low average density of the gas allows access to the radiative flux regime [34] with moderate laser energies ( $<1 \mathrm{~J}$ ) and relatively low shock velocities $\left(\approx 10 \mathrm{~km} \mathrm{~s}^{-1}\right)$. In Fig. 3 we present the plot representing the transitions between the various shock regimes for xenon plasmas. According to the figure and Ref. [34], the blast waves considered in this work are well within the radiative flux regime but well below the radiation dominated regime. Furthermore, according to Ref. [1], for the blast wave to be optically thin and radiative the mean free path of the photons, $\lambda_{\text {rad }}$, was calculated as

$\lambda_{\text {rad }}=\frac{1}{\rho k_{p}}$,

which must be larger than a characteristic size of the system, $h$, and the radiative cooling time, $\tau_{\text {rad }}$, must be shorter than the convective transport time, $t_{\text {conv }}=h / s$, where $s$ is the plasma sound speed. In Ref. [34] these comparisons were made for the xenon plasmas of the experiments, considering a characteristic size of $h=0.01 \mathrm{~cm}$. However, in that work the mean free path was calculated using the bremsstrahlung expression with

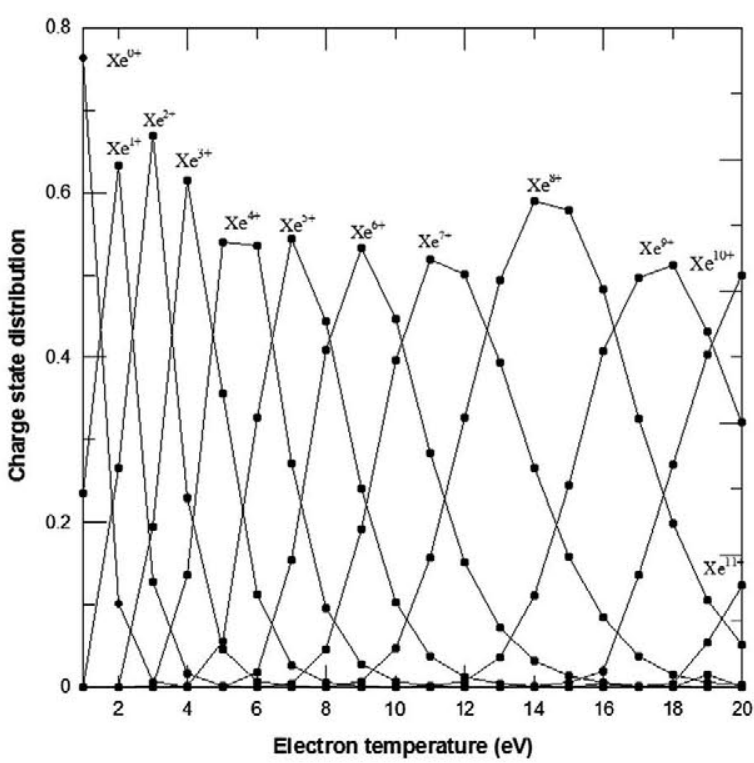

Fig. 2. CSD of xenon as a function of electron temperature for a density of matter of $5 \times 10^{-4} \mathrm{~g} \mathrm{~cm}^{-3}$.

no attempt to account for line contribution. This approximation is not accurate in the range of plasma conditions involved in this kind of experiments, since it was stated before the ionization of Xe is small and, therefore, the main contribution to the absorption comes from the line transition. According to Eq. (21), in this work we have calculated the PMFPs from the inverse of the Planck mean absorption coefficient including line, photoionization and bremsstrahlung contributions and the cooling function from CRSS calculations of the atomic level populations which are valid for the whole range of densities considered. The radiative cooling time is defined for optically thin plasmas as

$t_{\text {rad }}=2.4 \times 10^{-12} \frac{(\bar{Z}+1) T}{\bar{Z} n_{i} \Lambda}$,

where $A$ is the cooling function in ergs $\mathrm{cm}^{3} \mathrm{~s}^{-1}$, i.e. the radiative power loss is divided by the electron and ion 


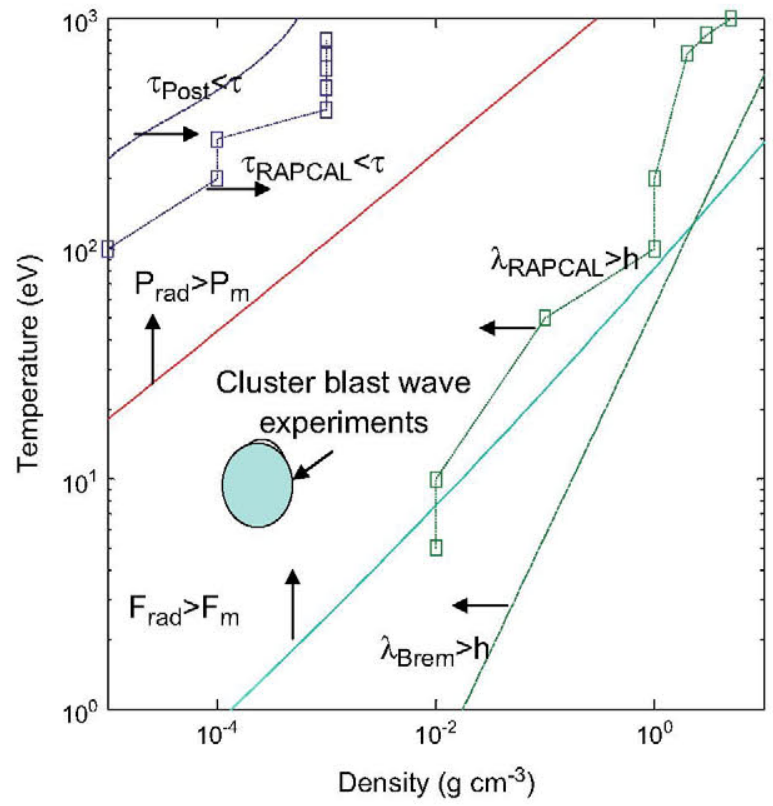

Fig. 3. Plot representing the various shock transitions regimes of Xe according to Refs. [1,64].

particle densities. The cooling time was calculated in Ref. [34] making use of the cooling function tabulated in Ref. [63] which is only accurate enough for low densities where Coronal equilibrium can be assumed. In Fig. 3 we show our calculations of both the cooling time and the PMFP and the ones provided in Ref. [34] in a wide range of matter densities and electron temperatures. It is observed that both calculations predict that the blast waves obtained in the experiments under analysis are optically thin and radiative, although we can detect that our calculations show that the region where the plasma can be assumed to be optically thin is shifted, for a given temperature, toward lower densities due to the increase of the opacity from line absorption. Obviously, this result should be only considered as an approached estimation of the character of the optical depth of the system. As it was concluded in [61], if we analyze the spectrally resolved opacities we detect that the plasma is optically thick for some ranges of photon energies, as we will show in next subsections, and that is the reason that a radiative precursor is detected in this kind of experiment [33-35]. Furthermore, from our calculations we find that for the density of $5 \times 10^{-4} \mathrm{~g} \mathrm{~cm}^{-3}$ the plasma enters in the radiative regime for temperatures lower than $\approx 300 \mathrm{eV}$. From the calculations using the cooling functions provided in Ref. [63], the temperature is around $600 \mathrm{eV}$. The cooling functions given in that work were calculated for electron densities lower than $10^{16} \mathrm{~cm}^{-3}$, whereas the electron density in this case is $\approx 10^{19} \mathrm{~cm}^{-3}$ and, therefore, their results could not be accurate. In any case, our calculations also predict that the blast waves under analysis are radiative.

\subsection{Analysis of the experiment}

In the rest of the paper we will analyze the experimental blast waves launched in xenon using the THOR laser system at

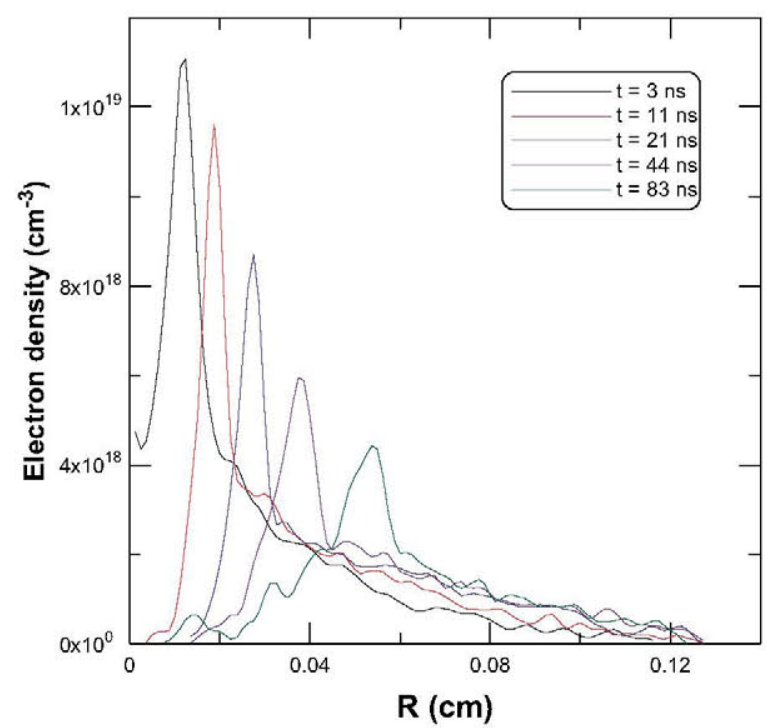

Fig. 4. Structure of the radiative blast wave as a function of the time.

the University of Texas [33]. The gas of xenon clusters was irradiated with a laser energy of $\approx 400 \mathrm{~mJ}$ at average gas density of $1.6 \times 10^{-4} \mathrm{~g} \mathrm{~cm}^{-3}$. The blast waves formed were characterized using time-resolved transverse interferometric and Schlieren imaging [34]. Fig. 4 shows typical electron density profiles for xenon cylindrical blast waves produced during this experiment at several times. The shock front is moving from the left to the right. From the figure, it is detected that a radiative precursor is ahead of the shock front. The average ionization values of pre-shock region are direct measurements from the interferograms since the ambient density of gas is known. Behind the shock the electron density is measured and the average ionization is calculated assuming a shell compression, defined as the ratio of the maximum mass density within the shell to the ambient gas density ( $c=\rho_{\text {shell }} / \rho_{\text {ambient }}$ ), equals to 2 [33]. This value for the compression was obtained from the ratio of the shell thickness to the shock front radius. This weak compression is because of the preheating of the gas ahead of the shock that reduces the Mach number [34].

On the other hand, the plasma temperature was not measured in this experiment, although in this kind of experiments this could be measured using X-ray Thomson scattering. This was estimated in Ref. [34] making use of an approached expression [64] derived from the Saha equation and thus assumes LTE. However LTE regime cannot be always assumed and therefore this estimation of the temperature could be not accurate enough and that could give, for example, unphysical solutions in the calculation of the compression ratio [34]. In the next subsections we present an estimation of the electron temperatures and we also perform an analysis of the shock shell and the radiative precursor; using the CRSS model implemented in ABAKO/RAPCAL.

\subsubsection{Estimation of the electron temperatures of the radiative blast wave}

In this work we have made an estimation of the electron temperature making use of the CRSS implemented in 
ABAKO/RAPCAL In particular, we performed a polynomial fitting, in density and temperature, of the plasma average ionization to a database generated with kinetics calculations of $A B A K O / R A P C A L$ enforcing a relative error lower than $0.1 \%$ [65]. Thus, the average ionization is given by

$\log \bar{Z}\left(n_{e}, T_{e}\right)=\sum_{i=0}^{n} \sum_{j=0}^{m} C_{i j}\left(\log n_{e}\right)^{i}\left(\log T_{e}\right)^{j}$

The coefficients of the fitting, $C_{i j}$, are determined by means of a Least Squares Regression. The maximum degree of the polynomial both in electron temperature, $m$, and density, $n$, was fixed to 7 in order to avoid oscillating behaviors. In general, it is impossible to find only one polynomial function to make the fit of the whole range of plasma conditions and this one must be divided into subsets to obtain a polynomial fitting in each subset. In this case, for the range of plasma conditions of interest in this work, the number of polynomial functions required has been 4 . Finally, in order to optimize the search of the subsets of plasma conditions and the corresponding polynomial functions a quad-tree algorithm was used. Therefore, using this fitting of the average ionization the electron temperature diagnosed is the one that, for a given electron density, provides an average ionization that matches with the experimental one, within a margin of error imposed, which was of $0.1 \%$ in this work.

In Fig. 4 we can observe small fluctuations due to the experimental measurement of the electron density. In order to avoid these fluctuations in the estimation of the electron temperature we have made a fitting of the electron densities using Gaussian functions. In Fig. 5 we present the resulting smoothing functions along with the representation of the average ionization, obtained from the smoothed densities, against the radial coordinate.

Finally, in Fig. 6 is shown the estimation of the electron temperature as a function of the radial coordinate for the times provided by the experiment. The largest temperature corresponds to the temperature of shock front. In this kind of experiments, due to the energy deposited by the laser, the largest electron temperature that can be reached is about $20 \mathrm{eV}$ and our numerical simulations of the temperature fulfill that restriction. The temperature of the shock front decays with time because of its loss of energy which is radiated away. This radiation ionizes the plasma ahead of the shock front and it is responsible for the origin of the radiative precursor. However, since this one is optically thin, the energy radiated is only partially absorbed by the radiative precursor, and eventually escapes. In any case the absorption would be more significant for the regions of the radiative precursor nearest to the shock front and, therefore, there is a diminution of the temperature as one moves away from the shock, as it can be observed in the figure. We can also observe from Figs. 4 and 6 that for the most distant points of the radiative precursor from

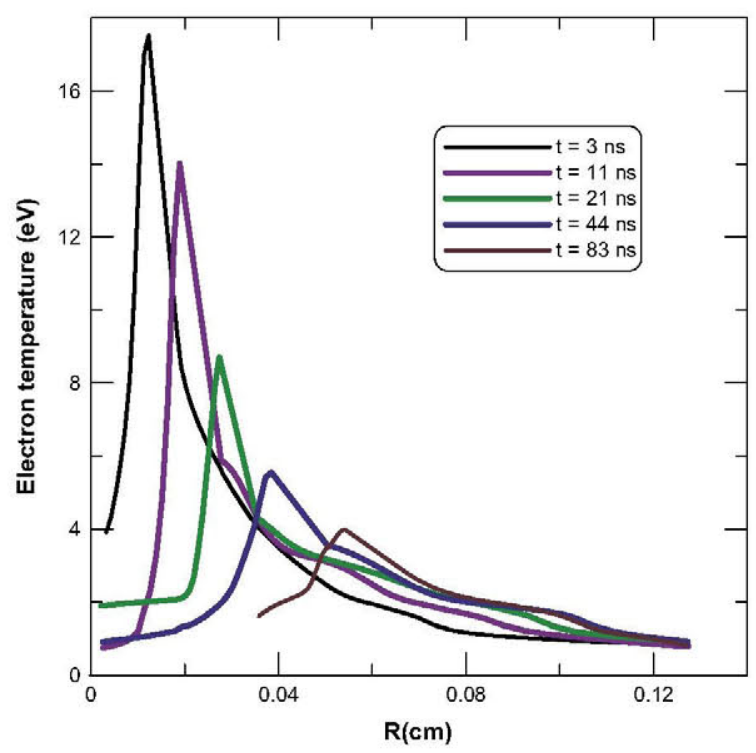

Fig. 6. Electron temperatures estimated using the CRSS model for the times provided by the experiment.
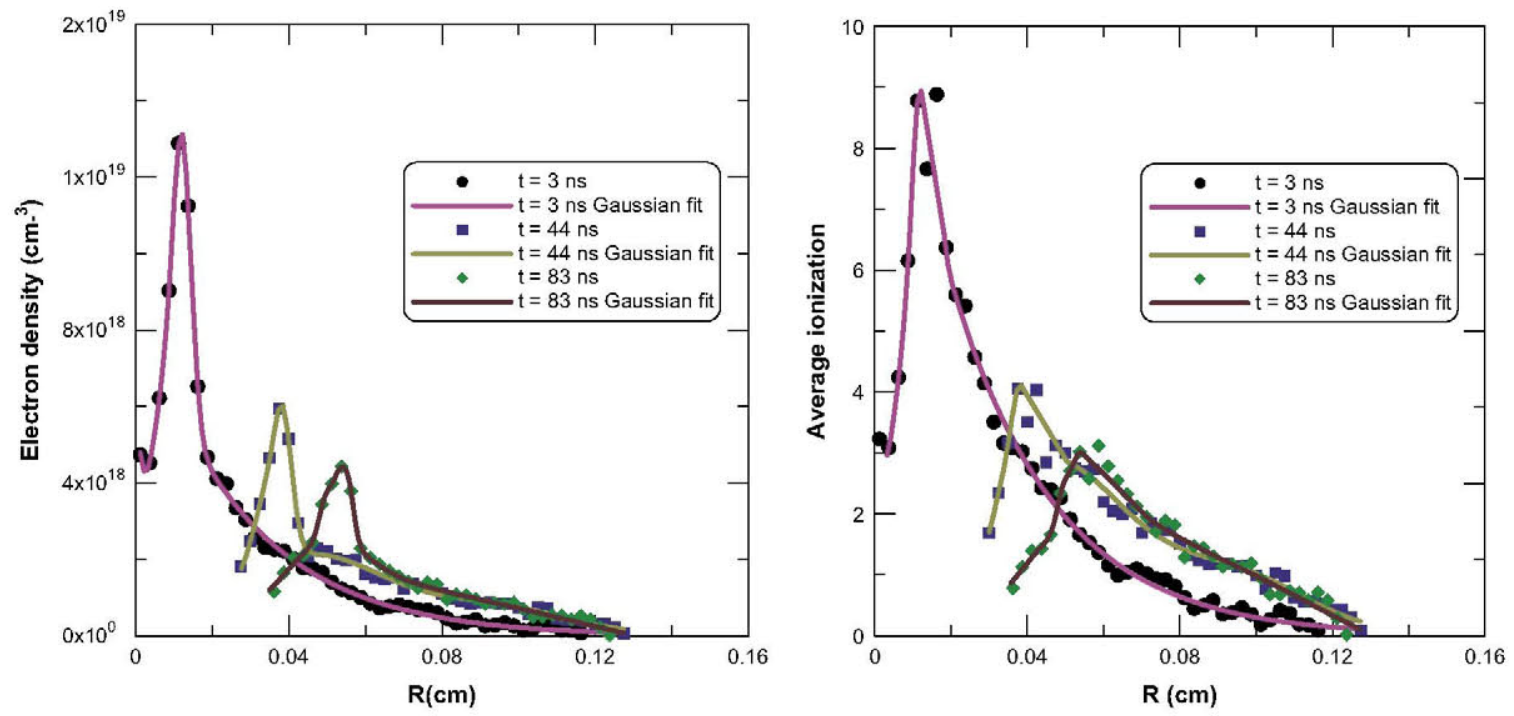

Fig. 5. Gaussian fit of the experimental electron density and average ionization for several times. 

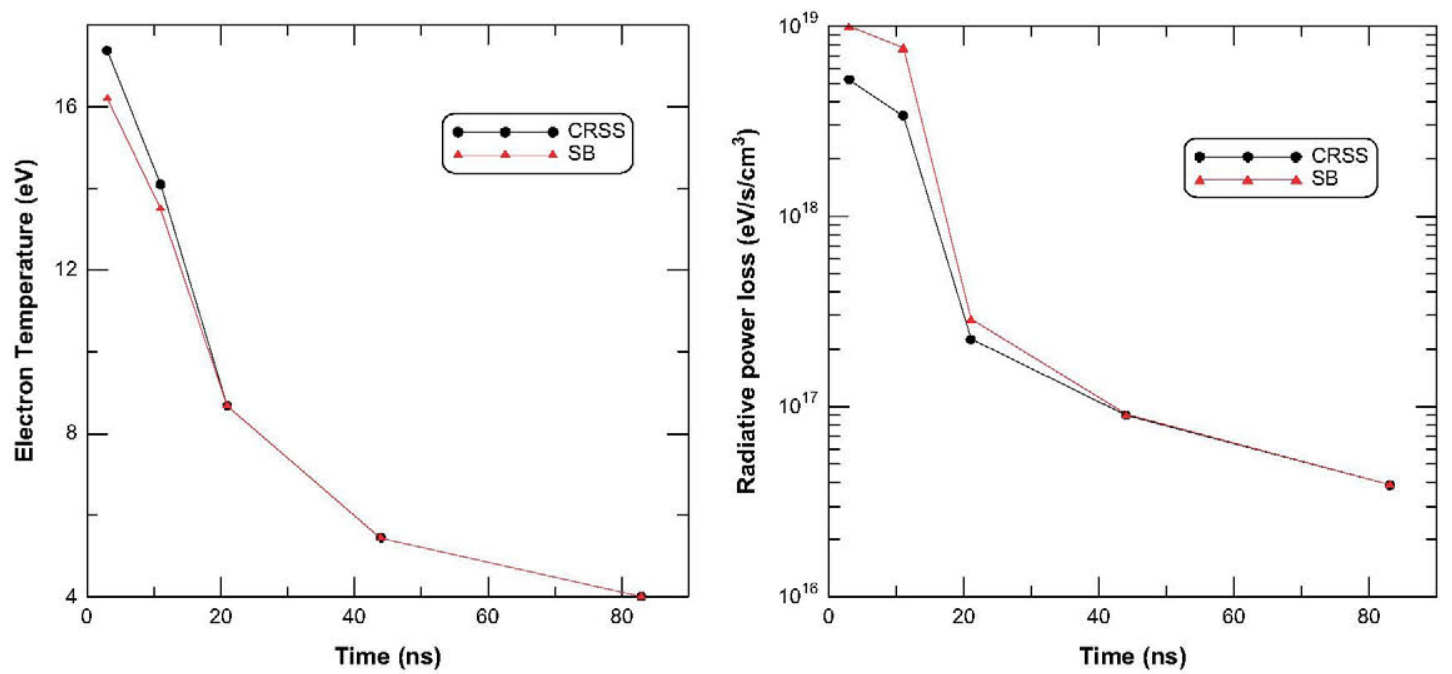

Fig. 7. Comparison between the CRSS and SB calculations of the shock temperatures (left) and radiative power losses (right) at different times.

the shock front $(R \geq 0.11 \mathrm{~cm})$ the electron densities and temperatures, and then the average ionization, do not change with time. Since the radiative precursor is mainly generated by the radiation, this result could mean that the same intensity arrives to this region of the radiative precursor at any time.

As said before, in Ref. [34] the temperature of the shock front was estimated assuming LTE. In Fig. 7 we have plotted the electron temperature for the shock front that calculated both using the CRSS and SB equations. From the figure it is observed that only for times later than $20 \mathrm{~ns}$ both calculations provide the same value of the electron temperature. On the other hand, for previous times some discrepancies are observed. For example, at 3 ns the CRRS and the SB estimations of the electron temperature are 17.38 and $16.20 \mathrm{eV}$, respectively, i.e. a relative error $\approx 7.3 \%$. In the figure we have also plotted the radiative power loss from the shock shell, which is a key magnitude in radiative shocks, calculated both using CRSS and SB equations. From the figure we detect that although at $20 \mathrm{~ns}$ the electron temperature estimated by both models are very similar that is not true for the radiative power loss. The models give at that time almost the same CDSs and, therefore, average ionizations and electron temperatures but the atomic levels are not in LTE and this is the reason of this discrepancy in the radiative power losses. Therefore, we can conclude that for times later than 40 ns a LTE simulation of the shock front could provide similar results than a CRSS simulation both in the estimation of the electron temperature and in the calculation of the radiative properties. However, from the figure we can observe that the temperature of the shock front changes appreciably with time, overall for times shorter than $20 \mathrm{~ns}$, and then, the stationary-state approach assumed in our NLTE simulation could be not accurate enough and a timedependent simulation could be needed. This fact could imply more differences between NLTE and LTE simulations and it is our aim to address this issue in a future work. Finally, from the figure of the radiative power loss we can observe that the shock shell radiates stronger at early

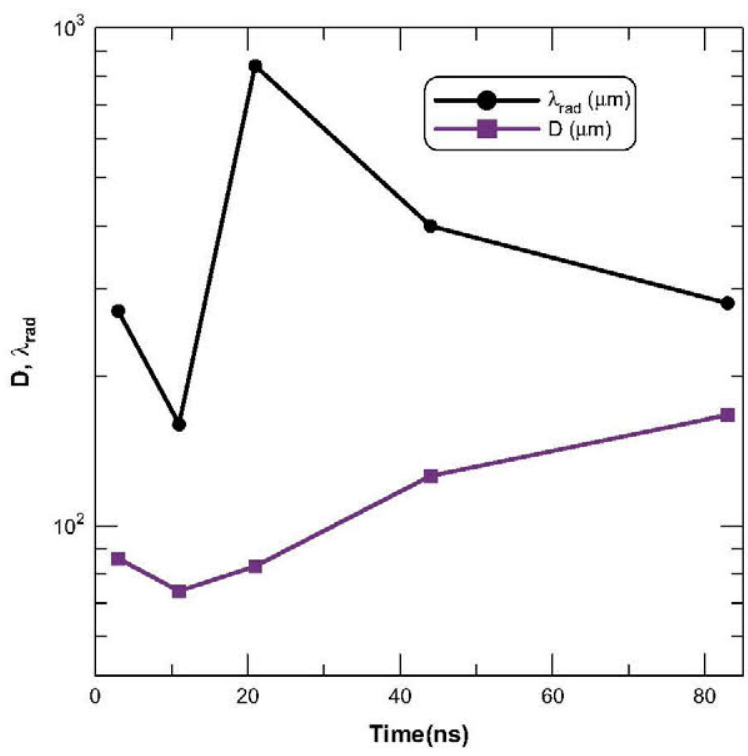

Fig. 8. Comparison between the PMFP and the FWHM (D) of the shock shell at different times.

times (until $\approx 20 \mathrm{~ns}$ ) and after that, since the shocked material is cooler, the radiative power loss diminishes. This fact agrees with the result obtained analyzing the evolution of the trajectory in which two phases can be distinguished [33]: (i) a strongly radiative one, up to $\approx 30 \mathrm{~ns}$, in which the estimated fraction of energy radiated to be between 90 and $100 \%$ of the incoming energy flux and (ii) at later times, where the radiative losses become less significant leading to an energy-conserving or gaining trajectory.

\subsubsection{Analysis of the plasma absorption in the shock shell}

Previously, it was commented that the type of experimental radiative blast wave considered in this work can be categorized as optically thin-thin. This conclusion was established by means of the comparison of the PMFP, 
calculated for a typical plasma condition in this kind of experiments, with a characteristic plasma length. In this section we have made a more detailed analysis of this assert for the experiment under analysis. As it is known, a comparison between the PMFP and the length of the plasma is an estimator of its optical behavior. In Fig. 8 we compare the PMFP of the shock shell with its fullwidth at half-maximum (FWHM) for the time instants under analysis. To calculate the PMFP we have assumed that the plasma in the shock shell is homogeneous with the temperature of the shock front. From the figure we observe that the PFMPs are always significantly larger than the FWHMs which is in line with the fact that the plasma in the shock shell could be considered as optically thin at any time.

We have also made an analysis of the opacity effects in the kinetics calculations, i.e. in obtaining the plasma level populations. If the plasma is optically thick, the set of the rate equations (Eq. (1)) must be solved together with the radiative transfer equation,

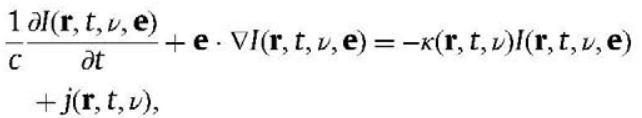

where $I$ is the specific intensity, $\nu$ the photon frequency and $\mathbf{e}$ a unitary vector in the direction of the radiation propagation. The emissivity and the absorption coefficient couple the radiative equation with the rate equations. In ABAKO it is assumed stationary conditions for the radiative transfer and, therefore, the first sum in the left hand side of Eq. (24) equals zero. In the current version of ABAKO only bound-bound opacity effects are taken into account. These ones are included in an approximate way by means of the escape factor formalism which avoids the explicit solution of the radiative transfer equation.

For a given line transition $\zeta i \leftrightarrow \zeta j$, the escape factor $\Lambda_{j i}$ is introduced as an alternative way of writing the net rate of line emission. The escape factors enter in the calculations in two ways. First, in the atomic physics calculations of excited-state populations. As a result there is an effective reduction in the Einstein spontaneous emission coefficient $A_{\zeta j \rightarrow \zeta i}$, which is written as $\Lambda_{j i} A_{\zeta j \rightarrow \zeta i}$. Second, they appear in the determination of the total emergent line intensity. This modification circumvents the need to perform a simultaneous calculation of radiation transport and atomic physics. To compute the escape factors we have adopted the technique described in [66]. Thus, assuming a uniform distribution of emitting atoms and isotropic emission, for the three basic geometries-plane, cylindrical and spherical-the escape factor $\Lambda_{j i}$ is written as

$\Lambda_{j i}=\int_{0}^{\infty} \phi_{i j}(\nu) \frac{1}{\tau_{i j}(\nu)} F\left[\tau_{i j}(\nu)\right] d \nu$.

$\tau_{i j}(\nu)=\kappa_{\zeta i \rightarrow \zeta_{j}}(\nu) L$ is the optical depth, where $L$ denotes the characteristic plasma dimension, i.e. slab width, cylinder or sphere radius. Finally, $F\left(\tau_{i j}\right)$ is a functional of the optical depth whose particular form depends on the considered geometry. For the cylindrical geometry $F(\tau)$ is computed by interpolation over a numerically defined function.

On the other hand, for each line transition, the escape factor depends implicitly on the populations of the lower,

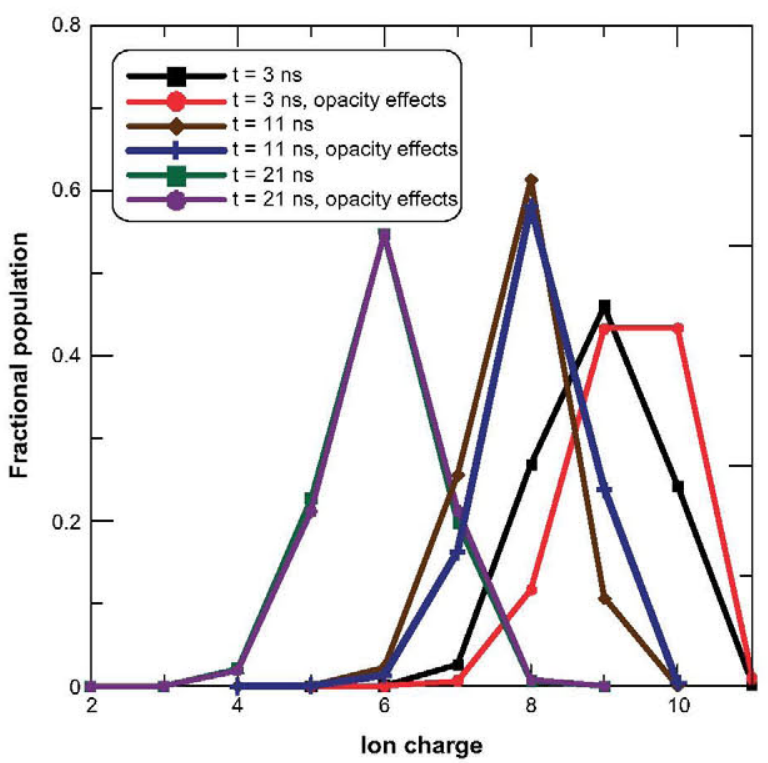

Fig. 9. Comparison between the CSDs of the shocked plasma calculated with and without opacity effects in the rate equation at three different times.

$N_{\zeta i}$, and upper level, $N_{\zeta j}$, since, according to Eq. (25), they are required to compute the absorption coefficient. Hence, in the case of optically thick plasmas, the system of rate equations must be solved iteratively until convergence is achieved within a prescribed tolerance. We have performed a kinetics calculation of the plasma in the shock shell including opacity effects. We have assumed that the plasma is homogeneous at the temperature of the shock front. Furthermore, we have supposed cylindrical geometry being the radius equals to the FWHM of the shock shell. In Fig. 9 we compare the CSDs calculated with and without opacity effects in the rate equations. From the figure we first observe that the CDS for times later than $20 \mathrm{~ns}$ is unaffected by the opacity effects, and, therefore, from a point of view of a kinetics calculation the plasma is optically thin for that times. At 3 and 11 ns we observe that the plasma self-absorption leads to an increase of the average ionization, which is the expected behavior. This effect is more significant at $3 \mathrm{~ns}$ than at $11 \mathrm{~ns}$ in which the fractional population of the most abundant ion is almost the same for both calculations (with or without selfabsorption). In any case, both at 3 and $11 \mathrm{~ns}$, the most abundant ions are the same in both simulations, and that means that the average ionizations will be quite similar. Thus, at 3 ns the average ionizations calculated assuming the plasma optically thin or optically thick are 9.32 and 8.92 , and at 11 ns 8.06 and 7.80, i.e. relative differences of $4.3 \%$ and $3.2 \%$ between both simulations at 3 and $11 \mathrm{~ns}$, respectively. We would like to point out that the real differences between optically thin and thick simulations would be lower since we have assumed for the optically thick simulations the highest electron temperature in the shock shell at each time, and that implies that, for a fixed density of matter, we are overestimating the selfabsorption effects. Even so, we have seen that these differences do not change appreciably the monochromatic 
emissivity and opacity, see Fig. 10. Thus, we can observe from the figure that the spectra are very similar and only the heights of some peaks are slightly decreased in the optically thick simulation, which is expected. Therefore, although at $3 \mathrm{~ns}$ and $11 \mathrm{~ns}$ some differences are detected in the kinetics calculations they are not important enough to introduce appreciable differences in the radiative properties, and then in a radiation-hydrodynamic simulation, and this result reinforced our view that the shock shell can be classified as optically thin at any time.

A further question is whether the self-absorption affects the intensity that propagates through the shock shell and which is eventually emitted. For this study we have assumed that the shock shell is a homogeneous medium in which the source function does not vary with the location, with planar geometry of thickness $D$. Then, the outgoing specific intensity of the shock front measured along the ray is given by

$I_{\nu}(D)=I_{\nu}(0) e^{-\tau_{\nu}(D)}+S_{\nu}\left(1-e^{-\tau_{\nu}(D)}\right)$,

with $S_{\nu}$ the source function and $\tau_{\nu}(D)$ the monochromatic optical thickness of a medium of total thickness $D$ measured along the beam given by

$\tau_{\nu}(D)=\int_{0}^{D} \kappa_{\nu}(s) d s$

For the intensity emitted from the shock shell we have assumed that $I_{\nu}(0)=0$. In Fig. 11 we show the calculations of the specific intensity at different times calculated including self-absorption or not, i.e. considering the shock shell as optically thin for the whole range of photon frequencies. From the figure it is observed that the selfabsorption in the shell reduces some of the main peaks of the intensity being that reduction more important for the early times. However, except in those peaks, in general the opacity effects do not change substantially the specific intensity that agrees with our conclusion that the shock shell could be considered as optically thin.

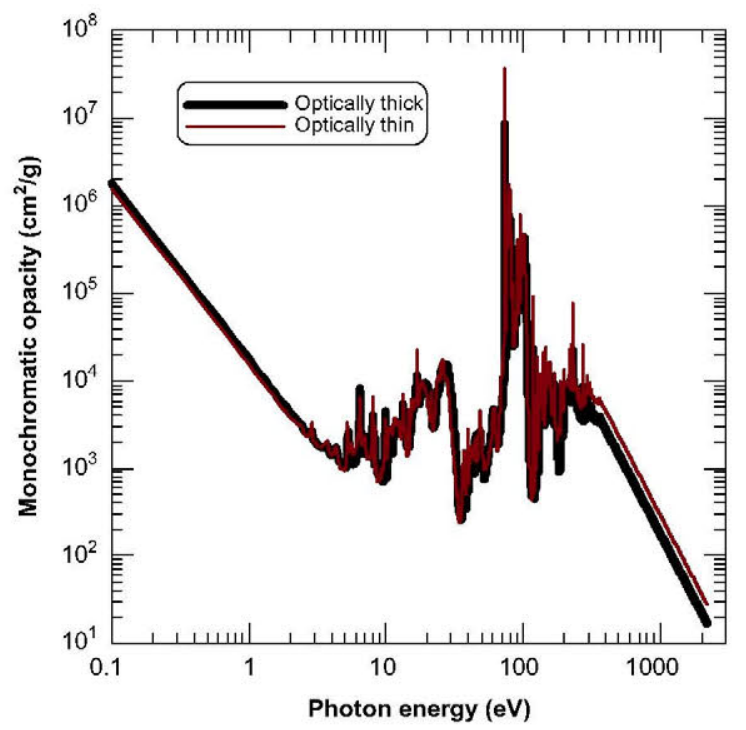

Fig. 10. Comparison between optically thin and thick calculations of the monochromatic opacity and emissivity of the shocked plasma at 3 ns.

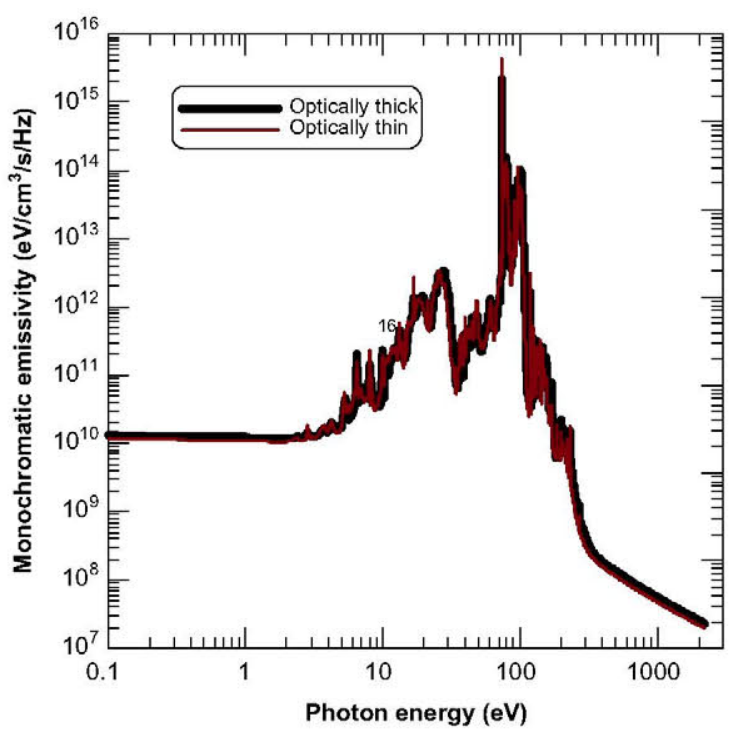

\subsubsection{Analysis of the absorption and emission of the radiative precursor}

In the previous section we concluded that the plasma in the shock shell could be considered as optically thin. The plasma in the radiative precursor has a lower density and temperature, and, therefore, self-absorption effects will be less important than in the shocked medium. So the plasma in the radiative precursor can also be considered as optically thin. However, its origin is the radiation emitted by the shocked medium and then, the radiative precursor should absorb some of this radiation. We have analyzed this absorption of the specific intensity. In order to make easier the analysis we have divided the radiative precursor in homogeneous layers characterized for average electron temperatures and densities. In Fig. 12 is shown the structure of layers considered at $3 \mathrm{~ns}$ and this is the one that we will analyze in the following. The electron temperatures of the radiative precursor were estimated using our CRSS and according to the thermodynamic regime map presented in Fig. 1, for the mass density of the radiative precursor $\left(1.6 \times 10^{-4} \mathrm{~g} \mathrm{~cm}^{-3}\right)$, LTE approach is accurate for temperatures lower than $\approx 8 \mathrm{eV}$. From Fig. 6 it is observed that the greatest temperatures reached in the radiative precursor are lower than $8 \mathrm{eV}$ and, therefore, LTE approach could be used for the estimation of the electron temperatures. However, as for the shocked shell, it is our aim to analyze time effects in the kinetics calculations, and then in the electron temperature estimation, in a future work. We will assume that the radiative precursor is already generated by the radiation escaped from the shock shell and we will study how the precursor absorbs the radiation that the shock front continues emitting at that time instant. The specific intensity is calculated using Eq. (26) but now $I_{\nu}(0) \neq 0$ For the first layer, $I_{\nu}(0)$ is the intensity coming from the shock front whereas for the rest of the layers denotes the outgoing intensity of the previous layer. In Fig. 13 we have represented the monochromatic optical depths of some layers of the radiative precursor at $3 \mathrm{~ns}$. A layer will be optically thick for optical depths greater than one. From the figure it is observed 

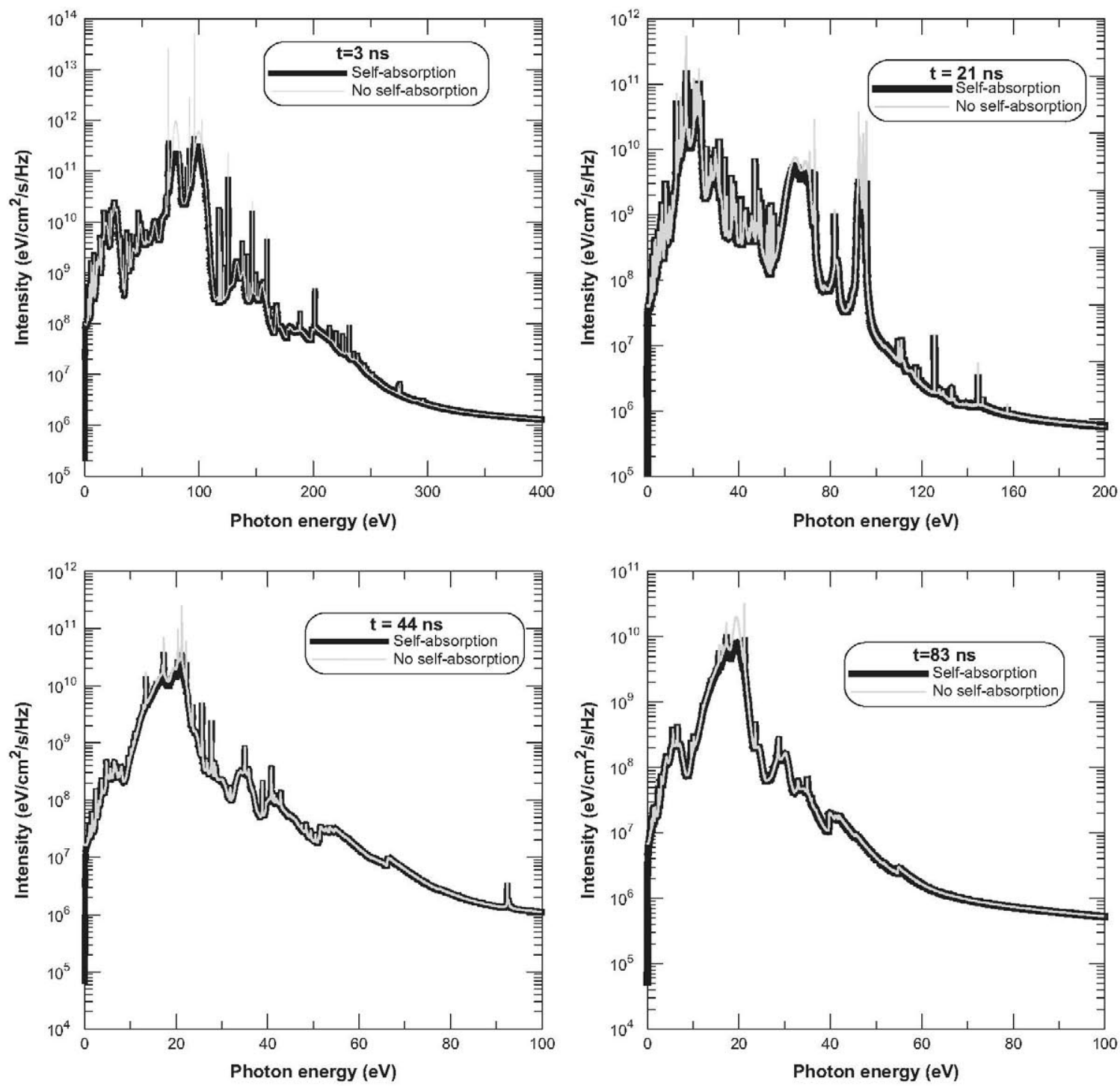

Fig. 11. Specific intensity from the shock shell at different times calculated including self-absorption or not.

that the layers of the radiative precursor are optically thin for almost the whole range of the photon energies involved except for some windows of energies where the criterion is not fulfilled. These windows are shifted towards lower photon energies as we move away from the shock front, i.e. as the number of the layer increases, what is expected since the average ionization decreases as the electron temperature also does. Thus, the layer 1 will mainly absorb the photons emitted by the shock front with energies around $20 \mathrm{eV}$ and $80 \mathrm{eV}$. In the layers 3 and 6 will be absorbed those with energies around $20 \mathrm{eV}$ whereas in the last layer the photons absorbed will have an energy around $10-15 \mathrm{eV}$. On the other hand, from the figure it is clear that those photons with energies higher than $100 \mathrm{eV}$ are not absorbed by the radiative precursor.

Finally, the radiative precursor will also emit radiation since it is ionized. We have checked the relative importance in the specific intensity of the self-emission of the radiative precursor with respect to the radiation coming from the shock shell. In Fig. 14 we have represented the outgoing specific intensity of some of the layers of the radiative precursor at $3 \mathrm{~ns}$ as well as the self-emission of the layer. With respect to influence of the self-emission of the radiative precursor in the intensity of the radiation that is propagating through it we can observe that this is relevant only for photon energies in the range $1-30 \mathrm{eV}$. However, this is the range in which the radiative precursor mainly absorbs and, therefore, the selfemission must be included in the simulation of the propagation of the intensity emitted by the shock through the radiative precursor.

\section{Conclusions}

In this work we have performed an analysis of some microscopic properties of radiative blast waves launched in xenon clusters. With this purpose we have employed the computational package ABAKO/RAPCAL designed to 


\begin{tabular}{cccccccc}
\hline Layer 1 & Layer 2 & Layer 3 & Layer 4 & Layer 5 & Layer 6 & Layer 7 & Layer 8 \\
$\left\langle\mathrm{T}_{\mathrm{e}}\right\rangle=7.55$ & $\left\langle\mathrm{~T}_{\mathrm{e}}\right\rangle=6.60$ & $\left\langle\mathrm{~T}_{\mathrm{e}}\right\rangle=5.50$ & $\left\langle\mathrm{~T}_{\mathrm{e}}\right\rangle=4.40$ & $\left\langle\mathrm{~T}_{\mathrm{e}}\right\rangle=3.50$ & $\left\langle\mathrm{~T}_{\mathrm{e}}\right\rangle=2.40$ & $\left\langle\mathrm{~T}_{\mathrm{e}}\right\rangle=1.40$ & $\left\langle\mathrm{~T}_{\mathrm{e}}\right\rangle=0.95$ \\
$\langle\overline{\mathrm{Z}}\rangle=5.60$ & $\langle\overline{\mathrm{Z}}\rangle=5.00$ & $\langle\overline{\mathrm{Z}}\rangle=4.40$ & $\langle\overline{\mathrm{Z}}\rangle=3.60$ & $\langle\overline{\mathrm{Z}}\rangle=2.90$ & $\langle\overline{\mathrm{Z}}\rangle=1.90$ & $\langle\overline{\mathrm{Z}}\rangle=0.80$ & $\langle\overline{\mathrm{Z}}\rangle=0.25$ \\
$\boldsymbol{D}=50$ & $\boldsymbol{D}=10$ & $\boldsymbol{D}=40$ & $\boldsymbol{D}=37$ & $\boldsymbol{D}=135$ & $\boldsymbol{D}=115$ & $\boldsymbol{D}=320$ & $\boldsymbol{D}=250$
\end{tabular}

Fig. 12. Division in layers of the radiative precursor at $t=3 \mathrm{~ns}$. The temperatures are in $\mathrm{eV}$ and the length of the layers in $\mu \mathrm{m}$.
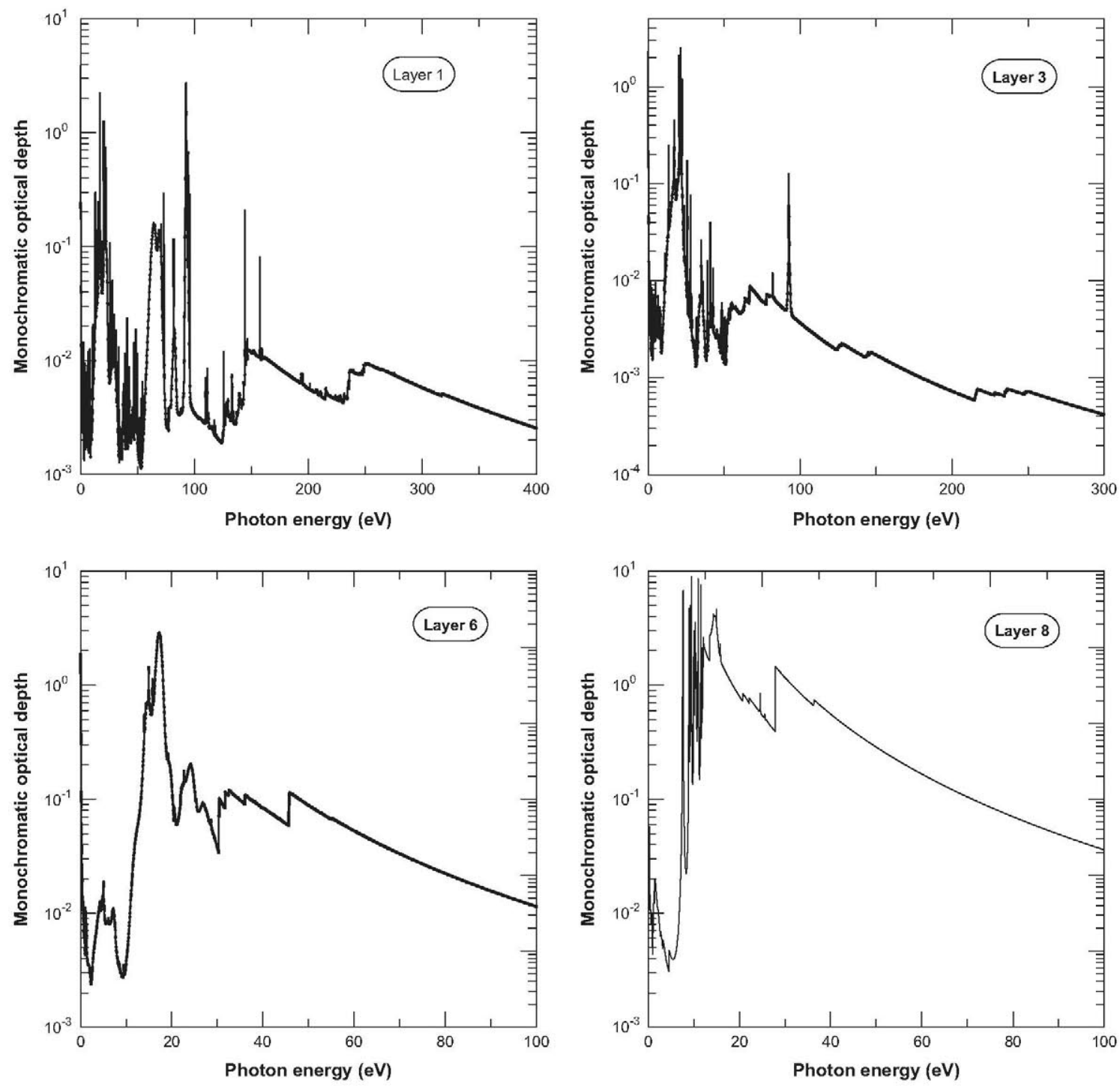

Fig. 13. Monochromatic optical depths of some layers of the radiative precursor at 3 ns.

make kinetics and radiative properties calculations both under LTE and NLTE approaches. In particular, we have studied the thermodynamic regimes in which these experiments are developed. We have found that, if we assume steady-state conditions, LTE approach would provide similar values of the radiative properties and average ionizations than those obtained under NLTE calculations for times later than $40 \mathrm{~ns}$ although if we are only interested in the determination of the average ionization and electron temperature this is valid for times later than $20 \mathrm{~ns}$. By means of the analysis of the radiative cooling time and the mean free path of the photons we have also proved that the blast waves obtained in the experiments are radiative and optically thin. Furthermore, for a particular experiment, we have made an estimation of the electron temperature of the shock shell and of the radiative precursor obtaining results that are compatible with the restriction of $20 \mathrm{eV}$ as the greatest temperature reached in this kind of experiments. For that experiment, we have also analyzed the effect of the absorption of radiation in the shock shell. We have found that selfabsorption effects in the kinetics calculations are not 

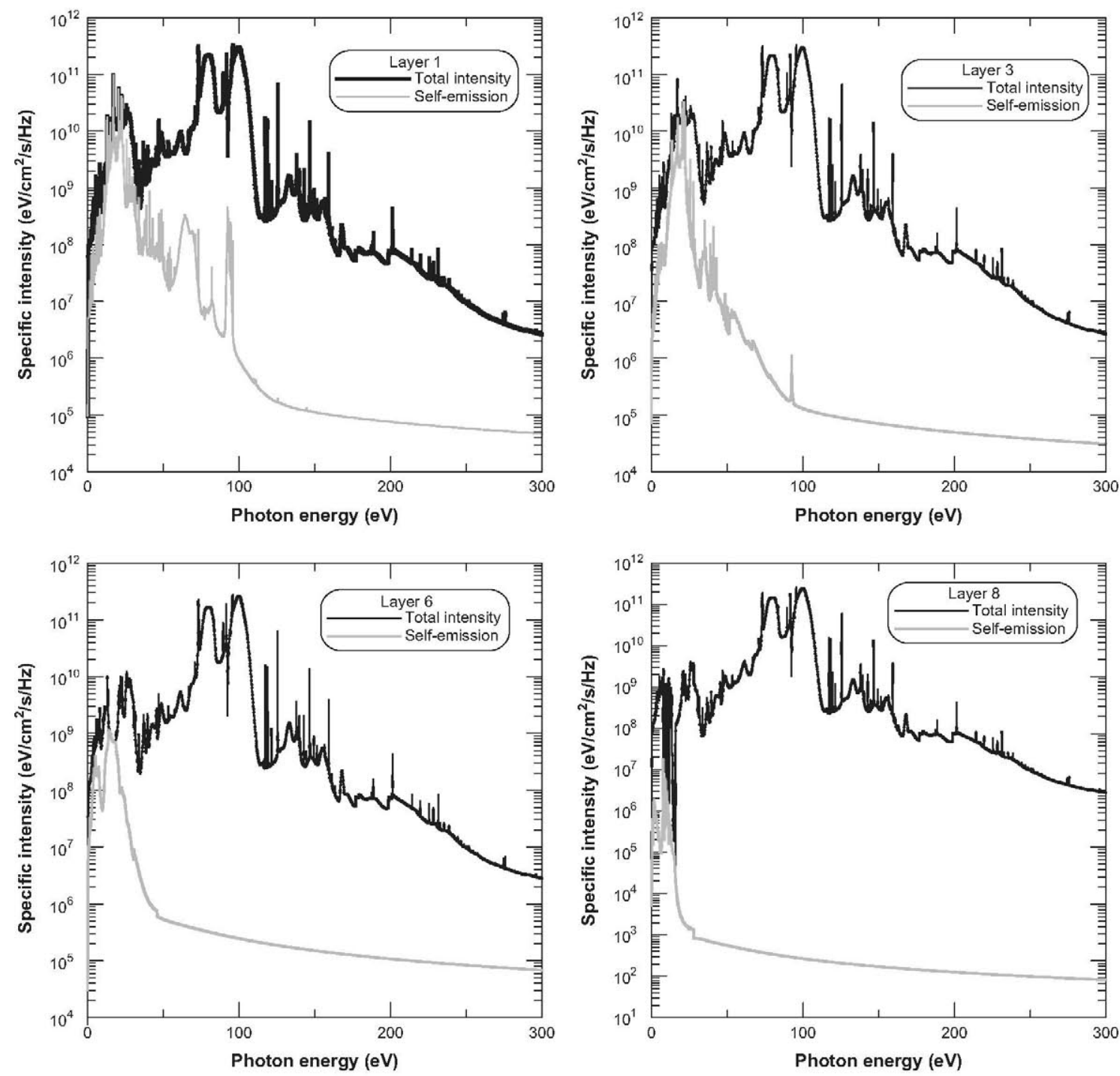

Fig. 14. Specific intensity and self-emission outgoing of some layers of the radiative precursor at $3 \mathrm{~ns}$.

important enough to modify the radiative properties and that only some peaks of specific intensity are affected by the self-absorption while the rest remains unaffected. We have also analyzed the absorption and the emission of the radiative precursor. With respect to the former, we have observed that the radiative precursor is transparent for photons with energies higher than $100 \mathrm{eV}$ and only photons with energies around $80 \mathrm{eV}$ (in the first layers) and $20 \mathrm{eV}$ (in all the layers) are absorbed. With respect to the influence of the self-emission in the intensity of the radiation that is going through the precursor, we have found that including self-absorption is important in the simulation of the propagation of the intensity emitted by the shock through the radiative precursor. Finally. as said before, all the calculations in this work were performed assuming stationary conditions in the kinetics model. It is our purpose in a future work to analyze the influence of time dependent kinetics calculations in these simulations.

\section{Acknowledgment}

This work has been supported by the Research Project of the Spanish Government ((ENE2009-11208/FTN) and by the Keep in touch Project of the European Union.

\section{References}

[1] Ryutov D, Drake RP, Kane J, Liang E, Remington BA, Wood-Vasey WM. Similarity criteria for the laboratory simulation of supernova hydrodynamics. Astrophys J 1999;518:821-32.

[2] Ryutov D, Drake RP, Remington BA. Criteria for scaled laboratory simulations of astrophysical MHD phenomena. Astrophys J Suppl Ser 2000;127:465-8.

[3] Remington BA, Drake RP, Ryutov D. Experimental astrophysics with high power lasers and Z pinches. Rev Mod Phys 2006;78:755-807.

[4] Castor Jl. Astrophysical radiation dynamics: the prospects for scaling. Astrophys Space Sci 2007;307:207-11.

[5] Falize E, Bouquet $S$, Michaut C. Scaling laws for radiating fluids: the pillar of laboratory astrophysics. Astrophys Space Sci 2009;322: 107-11. 
[6] Bouquet S, Falize E, Michaut C, Gregory CD, Loupias B, Vinci T, Koenig M. From lasers to the universe: scaling laws in laboratory astrophysics. High Energy Density Phys 2010;6:368-80.

[7] Moore AS, Lazarus J, Hohenberger M, Robinson JS, Gumbrell ET, Dunne $\mathrm{M}$, et al. Investigating the astrophysical applicability of radiative and non-radiative blast wave structure in cluster media. Astrophys Space Sci 2007;307:139-45.

[8] Drake RP. High energy density physics. Berlin: Springer-Verlag; 2005.

[9] Gonzalez M, Stehle C, Audit E, Busquet M, Rus B, Thais F, et al. Astrophysical radiative shocks: from modeling to laboratory experiments. Laser Part Beams 2006;24:535-40.

[10] Busquet M, Audit E, Gonzalez M, Stehle C, Thais F, Acef O, et al. Effect of lateral radiative loss on radiative shock propagation. High Energy Density Phys 2007;3:8-11.

[11] Stehle C, Ciardi A, Colombier JP, Gonzalez M, Lanz T, Marocchino A, et al. Scaling stellar jets to the laboratory: the power of simulations. Laser Part Beams 2009;27:709-17.

[12] Lindl J. Development of the indirect-drive approach to inertial confinement fusion and the target physics basis for ignition and gain. Phys Plasmas 1995;2:3933-4024.

[13] Symes DR, Hohenberger M, Doyle HW, Smith RA, Moore AS, Gumbrell ET, et al. Studying radiative shocks using laser driven blast waves in clustered gases. Nucl Instr Meth A 2011;653:186-8.

[14] Busquet M, Barroso P, Melse T, Bauduin D. Miniature shock tube for laser driven shocks. Rev Sci Instrum 2010;81:023502.

[15] Reighard AB, Drake RP, Dannenberg KK, Kremer DJ, Grosskopf M, Harding EC, et al. Phys Plasmas 2006;13:082901.

[16] Doss FW, Drake RP, Kuranz CC. Repeatability in radiative shock tube experiments. High Energy Density Phys 2010;6:157-61.

[17] Drake RP, Doss FW, McClarren RG, Adams ML, Amato N, Bingham D, et al. Radiative effects in radiative shocks in shock tubes. High Energy Density Phys 2011;7:130-40.

[18] Fryxell B, Rutter E, Myra ES. Simulations of laser experiments of radiative and non-radiative shocks. High Energy Density Phys 2012;8:141-9.

[19] Bouquet S, Stehle C, Koenig M, Chieze JP, Benuzzi-Mounaix A, Batani D, et al. Observation of laser driven supercritical radiative shock precursors. Phys Rev Lett 2004;22:225001.

[20] Koenig M, Vinci T, Benuzzi-Mounaix A, Ozaki N, Ravasio A, le Glohaec MR, et al. Radiative shocks: an opportunity to study laboratory astrophysics. Phys Plasmas 2006;13:056504.

[21] Benuzzi-Mounaix A, Koenig M, Ravasio A, Vinci T, Ozaki le N, Gloahec MR, et al. Laser-driven shock waves for the study of extreme matter states. Plasma Phys Control Fusion 2006;48:B347.

[22] Falize E, Ravasio A, Loupias B, Diziere A, Gregory CD, Michaut C, et al. High-energy density laboratory astrophysics studies of accretion shocks in magnetic cataclysmic variables. High Energy Density Phys 2012;8:1-4

[23] McClarren RG, Drake RP, Morel JE, Holloway JP. Theory of radiative shocks in the mixed: optically thick-thin case. Phys Plasmas 2010;17: 093301.

[24] Grun J, Stamper J, Manka C, Resnick J, Burris R, Crawford J, et al. Instability of Taylor-Sedov blast waves propagating through a uniform gas. Phys Rev Lett 1991;66:2738-41.

[25] Edens AD, Ditmire T, Hansen JF, Edwards MJ, Adams RG, Rambo PK, et al. Measurement of the decay rate of single-frequency perturbations on blast waves. Phys Rev Lett 2005;95:244503.

[26] Ditmire T, Shigemori K, Remington BA, Estabrook K, Smith RA. The production of strong blast waves through intense laser irradiation of atomic clusters. Astrophys J Suppl Ser 2000;127:299-304.

[27] Keilty KA, Liang EP, Ditmire T, Remington BA, Shigemori K, Rubenchik AM. Modeling of laser-generated radiative blast waves. Astrophys J 2000;538:645-52.

[28] Shigemori K, Ditmire T, Remington BA, Yanovsky V, Ryutov D, Estabrook $\mathrm{KG}$, et al. Developing a radiative shock experiment relevant to astrophysics. Astrophys J 2000;533:L159-62.

[29] Edwards MJ, MacKinnon AJ, Zweiback J, Shigemori K, Ryutov D, Rubenchik AM, et al. Investigation of ultrafast laser-driven radiative blast waves. Phys Rev Lett 2001;87:085044.

[30] Moore AS, Symes DR, Smith RA. Tailored blast wave production pertaining to supernova remnants. Phys Plasmas 2005;12:052707.

[31] Symes DR, Osterhoff J, Faustlin R, Maurera M, Bernstein AC, Moore AS, et al. Production of periodically modulated laser-driven blast waves in a clustering gas. High Energy Density Phys 2007;3:353-7.

[32] Moore AS, Gumbrell ET, Lazarus J, Hohenberger M, Robinson JS, Smith RA, et al. Full-trajectory diagnosis of laser-driven radiative blast waves in search of thermal plasma instabilities. Phys Rev Lett 2008; 100:055001.

[33] Osterhoff J, Symes DR, Edens AD, Moore AS, Hellewell E, Ditmire T. Radiative shell thinning in intense laser-driven blast waves. New Phys 2009; 11:023022.
[34] Symes DR, Hohenberger M, Lazarus J, Osterhoff J, Moore AS, Fustin $\mathrm{RR}$, et al. Investigations of laser-driven radiative blast waves in clustered gases. High Energy Density Phys 2010;6:274-9.

[35] Hohenberger M, Symes DR, Lazarus J, Doyle HW, Carley RE, Moore AS, et al. Observation of a velocity domain cooling instability in a radiative shock. Phys Rev Lett 2010;105:205003.

[36] Robbie SIO, Doyle HW, Symes DR, Smith RA. A study of ambient upstream material properties using perpendicular laser driven radiative blast waves in atomic cluster gases. High Energy Density Phys 2012;8:55-9.

[37] Chevalier RA, Imamura JN. Linear analysis of an oscillatory instability of radiative shock waves. Astrophys J 1982;261:543-9.

[38] Rodriguez R, Florido R, Gil JM, Rubiano JG, Suarez D, Martel P, et al. Collisional-radiative calculations of optically thin and thick plasmas using the computational package ABAKO/RAPCAL. Commun Comput Phys 2010;8:185-210.

[39] Florido R, Rodriguez R, Gil JM, Rubiano JG, Martel P, Minguez E, et al. Modeling of population kinetics of plasmas that are not in local thermodynamic equilibrium, using a versatile collisionalradiative model based on analytical rates. Phys Rev E 2009;80: 056402 .

[40] Rodriguez R, Florido R, Gil JM, Rubiano JG, Martel P, Minguez E. RAPCAL code: a computational package to compute radiative properties for optically thin and thick low and high-Z plasmas in a wide range of density and temperature. Laser Part Beams 2008;26: $433-48$

[41] Bowen C, Lee RW, Ralchenko Yu. Comparing plasma population kinetics codes: review of the NLTE-3 Kinetics Workshop. J Quant Spectrosc Radiat Transfer 2006;99:102-19.

[42] Rubiano JG, Florido R, Bowen C, Lee RW, Ralchenko Y. Review of the 4th NLTE code comparison workshop. High Energy Density Phys 2007;3:225-32.

[43] Fontes CI. Abdallah J, Bowen C, Lee RW, Ralchenko Y. Review of the NLTE-5 kinetics workshop. High Energy Density Phys 2009;5:15-22.

[44] Lotz W. Electron-impact ionization cross-sections and ionization rate coefficients for atoms and ions from hydrogen to calcium. Z Phys 1968;206:241-7.

[45] Van Regemorter HV. Rate of collisional excitation in stellar atmospheres. Astrophys J 1962;136:906-15.

[46] Kramers HA. On the theory of X-ray absorption and of the continuous X-ray spectrum. Philos Mag 1923;46:836-71.

[47] Griem HR. Principles of plasma spectroscopy. Cambridge: Cambridge University Press; 1997.

[48] Gu MF. The flexible atomic code. Can J Phys 2008;86:675-89.

[49] Bauche J, Bauche-Arnoult V, Klapisch M. Transition arrays in the spectra of ionized atoms. Adv At Mol Phys 1987;23:131-95.

[50] Stewart JC, Pyatt KD. Lowering of ionization potentials in plasmas. Astrophys J 1966;144:1203-11.

[51] Hansen S, Bauche J, Bauche-Arnoult C, Gu MF. Hybrid atomic models for spectroscopic plasma diagnostics. High Energy Density Phys 2007;3:109-14

[52] Peyrusse O, Bauche-Arnoult C, Bauche C. Effective superconfiguration temperature and the radiative properties of nonlocal thermodynamical equilibrium hot dense plasma. Phys Plasmas 2005;12: 063302.

[53] Cowan RD. The theory of atomic structure. Berkeley: University of California Press; 1981.

[54] Dimitrijevic MS, Konjevic N. Simple estimates for Stark-broadening of ion lines instellar plasmas. Astron Astrophys 1987;172:345-9.

[55] Rose SJ. Calculations of the radiative opacity of laser-produced plasmas. J Phys B 1992;25:1667-81.

[56] Rutten RJ. Radiative transfer in stellar atmospheres. In: Lectures notes, 8th ed. Utretch: Utretch University; 2003.

[57] Serduke FJD, Minguez E, Davidson SJ, Iglesias CA. WorkOp-IV summary: lessons from iron opacities. J Quant Spectrosc Radiat Transfer 2000;65:527-41

[58] Chung HK, Fournier KB, Lee RW. Non-LTE kinetics modeling of krypton ions: calculations of radiative cooling coefficients. High Energy Density Phys 2006;2:7-15.

[59] Karzas WJ, Latter R. Electron radiative transitions in a Coulomb field. Astrophys J 1961;6:167-212.

[60] Rodriguez R, Gil JM, Florido R, Rubiano JG, Mendoza MA, Martel P et al. Determination and analysis of the thermodynamic regimes of xenon plasmas. Contrib Plasma Phys 2011;51:863-76.

[61] Rodriguez R, Gil JM, Florido R, Rubiano JG, Mendoza MA, Martel P, et al. Determination of the average ionization and thermodynamic regimes of xenon plasmas with an application to the characterization of blast waves launched in xenon clusters. High Energy Density Phys 2011;7:71-6. 
[62] Bouquet S, Teyssier R, Chieze JP. Analytical study and structure of a stationary radiative shock. Astrophys J Suppl Ser 2000;127:245-52.

[63] Post DE, Jensen RV, Tarter CB, Gransberger WH, Lokke WA. Steadystate radiative cooling rates for low-density high-temperature plasmas. Atom Data Nucl Tables 1977;20:397-439.

[64] Drake RP. Radiative shocks in astrophysics and the laboratory. Astrophys Space Sci 2005;298:49-59.
[65] Rodriguez R, Gil JM, Espinosa G, Florido R, Rubiano JG, Mendoza MA, et al. Determination and analysis of plasma parameters for simulations of radiative blast waves launched in clusters of xenon and krypton. Plasma Phys Control Fusion 2012;54:045012.

[66] Mancini RC, Joyce RF, Hooper Jr. CF. Escape factors for Starkbroadened line profiles. J Phys B 1987;20:2975-87. 